\title{
Neuropsychological Impairments and Their Cognitive Architecture in Mild Cognitive Impairment (MCI) with Lewy Bodies and MCI-Alzheimer's Disease
}

\author{
Joanna Ciafone ${ }^{1}$ (1) , Alan Thomas ${ }^{1}$, Rory Durcan ${ }^{1}$, Paul C Donaghy ${ }^{1}$, Calum A Hamilton ${ }^{1}$, Sarah Lawley ${ }^{1}$, Gemma Roberts ${ }^{1,2}$, \\ Sean Colloby ${ }^{1}$, Michael J Firbank ${ }^{1}$, Louise Allan ${ }^{3}$, George Petrides ${ }^{2}$, John-Paul Taylor ${ }^{1}$, John T O’Brien ${ }^{4}$ and Peter Gallagher ${ }^{1}$ ** \\ ${ }^{1}$ Translational and Clinical Research Institute, Faculty of Medical Sciences, Newcastle University, United Kingdom \\ ${ }^{2}$ Nuclear Medicine Department, Royal Victoria Infirmary, Newcastle upon Tyne, United Kingdom \\ ${ }^{3}$ University of Exeter Medical School, South Cloisters, University of Exeter, United Kingdom \\ ${ }^{4}$ Department of Psychiatry, University of Cambridge School of Clinical Medicine, Cambridge, United Kingdom
}

(Received April 20, 2021; Final Revision July 27, 2021; Accepted August 13, 2021; First Published Online October 20, 2021)

\begin{abstract}
Objective: The present study aimed to clarify the neuropsychological profile of the emergent diagnostic category of Mild Cognitive Impairment with Lewy bodies (MCI-LB) and determine whether domain-specific impairments such as in memory were related to deficits in domain-general cognitive processes (executive function or processing speed). Method: Patients $(n=83)$ and healthy age- and sex-matched controls $(n=34)$ underwent clinical and imaging assessments. Probable MCI-LB $(n=44)$ and MCI-Alzheimer's disease (AD) $(n=39)$ were diagnosed following National Institute on Aging-Alzheimer's Association (NIA-AA) and dementia with Lewy bodies (DLB) consortium criteria. Neuropsychological measures included cognitive and psychomotor speed, executive function, working memory, and verbal and visuospatial recall. Results: MCI-LB scored significantly lower than MCI-AD on processing speed [Trail Making Test B: $p=.03, g=.45$; Digit Symbol Substitution Test (DSST): $p=.04, g=.47$; DSST Error Check: $p<.001, g=.68$ ] and executive function [Trail Making Test Ratio (A/B): $p=.04, g=.52$ ] tasks. MCI-AD performed worse than MCI-LB on memory tasks, specifically visuospatial (Modified Taylor Complex Figure: $p=.01, g=.46$ ) and verbal (Rey Auditory Verbal Learning Test: $p=.04, g=.42$ ) delayed recall measures. Stepwise discriminant analysis correctly classified the subtype in $65.1 \%$ of MCI patients (72.7\% specificity, $56.4 \%$ sensitivity). Processing speed accounted for more group-associated variance in visuospatial and verbal memory in both MCI subtypes than executive function, while no significant relationships between measures were observed in controls (all $p \mathrm{~s}>.05$ ) Conclusions: MCI-LB was characterized by executive dysfunction and slowed processing speed but did not show the visuospatial dysfunction expected, while MCI-AD displayed an amnestic profile. However, there was considerable neuropsychological profile overlap and processing speed mediated performance in both MCI subtypes.
\end{abstract}

Keywords: Cognitive Dysfunction, Cognition, Learning, Executive Function, Lewy Body Disease, Dementia

\section{INTRODUCTION}

Mild Cognitive Impairment (MCI) may be conceptualized as an intermediate stage between dementia and healthy ageing in which activities of daily living are preserved (Arnáiz \& Almkvist, 2003). While most commonly associated with Alzheimer's disease (AD), MCI can be caused by other diseases and evidence-based recommendations for diagnosis of

*Correspondence and reprint requests to: Peter Gallagher, Translational and Clinical Research Institute, Faculty of Medical Sciences, Newcastle University, Newcastle upon Tyne, NE2 4HH, United Kingdom. Telephone: +44 (0)191 208 7166, Fax: +44 (0)191 208 5227, E-mail: peter.gallagher@newcastle.ac.uk prodromal dementia with Lewy bodies (MCI-LB) have recently been published (McKeith et al., 2020). Neuropsychological impairments in dementia with Lewy bodies (DLB) and AD differ, but few well-controlled comparative studies of sufficient sample size have been done in the MCI stages (see Ciafone, Little, Thomas, \& Gallagher, 2020; Hemminghyth, Chwiszczuk, Rongve, \& Breitve, 2020 for reviews). In MCI-LB, deficits have been reported predominantly in domains impaired in DLB (i.e., visuospatial function, attention, executive function; Donaghy, Taylor, et al., 2018; Donaghy, O'Brien, \& Thomas, 2015) although other studies suggest a broad (Kemp et al., 2017) and heterogeneous (Hemminghyth et al., 2020) range of cognitive deficits. 
In a recent review, a third of neuropsychological outcome variables from six primary studies differed significantly between MCI-LB and MCI due to AD, suggesting possible dissociation, although most studies were small or lacked biomarker data to support diagnoses (Ciafone et al., 2020). Verbal learning and memory appeared less affected in MCI-LB (Ciafone et al., 2020) in line with AD's pronounced memory encoding deficits (Lange et al., 2002; Martin, Brouwers, Cox, \& Fedio, 1985). However, when compared to matched test norms, some studies suggested that substantial numbers of MCI-LB present with verbal memory impairment (Ferman et al., 2013; Kemp et al., 2017), indicating amnesia should not be taken as a reliable discriminator of prodromal AD. Memory impairment is especially present in DLB patients with markers of concurrent AD-type pathology using cerebrospinal fluid (CSF) or post-mortem neuropathology (Howard et al., 2021; Lemstra et al., 2017). There was also heterogeneity within domains: subtypes generally differed on some but not all measures of a given domain within a single study (Ciafone et al., 2020).

In cognitive psychology, multicomponential approaches fractionate the cognitive system into "domain-specific" components with separate functions and capacities (e.g., verbal and visuospatial) and "domain-general" resources, such as processing speed and executive functions (Logie, 2011). Components work in parallel to complete complex tasks and scaffold memory performance (Brown \& Wesley, 2013). Neuropsychological studies of hierarchical cognitive organization, as well as prominent resource models of agerelated cognitive decline, postulate that impairments observed in domain-specific tasks, for example, delayed verbal or visuospatial memory, can be the secondary result of domain-general, primary impairments (Kemp et al., 2017; Luszcz \& Bryan, 1999; MacDonald, Hultsch, Strauss, \& Dixon, 2003). Executive function and processing speed have both been proposed as such explanatory domain-general impairments in aging and disease. Consequently, memory deficits in MCI-LB may be underpinned by core, "domaingeneral" processing dysfunction, in contrast to the memory storage impairments characteristic of MCI-AD (Ciafone et al., 2020; Jicha et al., 2010; McKeith et al., 2020). DLB is associated with slower processing speed than mild $A D$ (Breitve et al., 2018; Cagnin et al., 2015), and the largest overall deficits in MCI-LB were recently reported as processing speed and executive function (Kemp et al., 2017).

There have been few studies of the neuropsychology of clinically-defined MCI-LB when compared to MCI-AD and, to our knowledge, no prospective studies of MCI-LB and healthy comparison subjects that use biomarkers identified in recent consensus diagnostic criteria. The present study was designed to examine the neuropsychological profile of MCI-LB compared with MCI-AD and healthy older people in a comprehensively assessed prospective cohort, with diagnosis supported by two validated biomarkers of Lewy body disease and in line with the recently published MCI-LB research criteria (McKeith et al., 2020). Specifically, we hypothesized: 1) greater deficits in visuospatial and executive function and slowed speed of processing in MCI-LB relative to MCI-AD and controls; 2) poorer performance by MCI-AD relative to MCI-LB and controls in delayed verbal recall, in line with the amnestic profile of $\mathrm{AD}$; and 3) domain-specific neuropsychological impairments in MCI are underpinned by the domain-general processing resources of executive function and processing speed.

\section{METHOD}

\section{Participants, diagnosis, and clinical assessments}

Patients over 60 years old with a clinical diagnosis of MCI in Memory Services were recruited in the north east of England. Control participants were recruited from relatives, friends, and a volunteer database and matched overall to the MCI groups on age. Patients were identified who had symptoms which may be related to prodromal DLB, such as autonomic, visual or olfactory disturbances, or any indications of core features of DLB. Participants were excluded if there was evidence of clinical stroke or frontotemporal atrophy on magnetic resonance imaging (MRI), Parkinson's disease (PD) established at least a year before cognitive decline, or severe mental illness (current major depression, bipolar disorder, schizophrenia). The study received ethical approval from the National Research Ethics Service Committee North East-Newcastle \& North Tyneside 2 (Research Ethics Committee Identification Number 15/NE/0420). Subjects were provided written informed consent after receiving a complete description of the study and were treated in accordance with the ethical standards of the Helsinki Declaration.

After consent all participants underwent a research clinical diagnostic assessment and neurological examination by a medical doctor (RD, SL) and were offered imaging for biomarkers ( ${ }^{123}$ I-FP-CIT SPECT, cardiac MIBG) as detailed elsewhere (Firbank et al., 2020; Roberts et al., 2020). FPCIT and MIBG scans were rated blind to clinical information. All had MRI brain scans which were consistent with their diagnoses. At the time of the scans and clinical assessment the Movement Disorder Society (MDS) Unified Parkinson's Disease Rating Scale (UPDRS-III; Goetz et al., 2008) motor subsection, Epworth Sleepiness Scale (ESS; Johns, 1991), and Geriatric Depression Scale (GDS; D'Ath, Katona, Mullan, Evans, \& Katona, 1994) were administered to study subjects. The Instrumental Activities of Daily Living (IADL; Lawton, Brody, \& Médecin, 1969) scale, North-East Visual Hallucinations Inventory (NEVHI; Mosimann et al., 2008), Neuropsychiatric Inventory (NPI; Cummings et al., 1994), Clinician Assessment of Fluctuation (CAF; Walker et al., 2000), and Dementia Cognitive Fluctuation Scale (DCFS; Lee et al., 2014) were administered to informants of patients. Clinical Dementia Rating scale (CDR; Hughes, Berg, Danziger, Coben, \& Martin, 1982) and Cumulative Illness Rating Scale for Geriatrics (CIRSG; Miller et al., 1992) were completed on the basis of the clinical history and other research assessments. Premorbid IQ was estimated using the National Adult Reading Test 
(Nelson \& Willison, 1991). All participants were reviewed annually at which the clinical diagnostic assessments and rating scales were repeated.

As detailed elsewhere (Thomas et al., 2019), at baseline and annually a three-person consensus clinical panel of experienced Board Certified old age psychiatrists (AJT, PCD, JPT) independently reviewed clinical notes taken from the research assessment and confirmed diagnoses. This consensus panel method has previously been validated against autopsy and is recognized by regulatory authorities as the clinical gold standard for living patients (McKeith et al., 2000; McKeith et al., 2007). A diagnosis of probable MCILB was given if a patient had two or more core Lewy body symptoms (with positive or negative scan results) or one core symptom in addition to a positive FP-CIT or MIBG scan, in accordance in the current consensus research criteria for diagnosis of probable MCI-LB (McKeith et al., 2020). Patients meeting criteria for possible MCI-LB (one core symptom or just one abnormal scan) were not included in this analysis. The clinical diagnosis of MCI-AD was made as set out in the criteria from Albert et al. (2011). Firstly, subjective and objective cognitive decline consistent with $\mathrm{AD}$ were established, along with generally maintained independence of function in everyday life. As directed in the criteria, other causes were then excluded including evidence of vascular cognitive impairment, primary progressive aphasia, and behavioural variant FTD, along with Lewy body disease. We had access to participant medical records and imaging results with their consent, allowing us to identify and exclude cases of probable vascular cognitive impairment.

Control subjects were free of subjective memory complaints or such concerns from others, evidence of any movement disorder, were not on antidementia or anti-PD drugs, and had Mini-Mental State Examination (MMSE) $\geq 26$ and normal MRI scans. The present study reports comparison of probable MCI-LB (henceforth MCI-LB) with MCI-AD and controls, in line with previous work in dementia which has compared probable DLB with AD dementia.

\section{Neuropsychological assessment}

A comprehensive neuropsychological assessment was administered by a trained researcher in participant homes or a clinical research facility over the course of two or more days. Tasks included the Addenbrooke's Cognitive Examination-Revised (ACE-R; Mioshi, Dawson, Mitchell, Arnold, \& Hodges, 2006), a 100-point cognitive screening test from which MMSE (Folstein, Folstein, \& McHugh, 1975) score was derived, Corsi Blocks (Corsi, 1972), a computerized adaptation of the Visual Patterns Task (VPT; Della Sala, Baddeley, Gray, \& Wilson, 1997), Modified Taylor Complex Figure (MTCF; Hubley \& Tremblay, 2002), Graded Naming Test (GNT; Warrington, 1997), Rey Auditory Verbal Learning Test [RAVLT; outcome measures: Maximum (most words recalled in any trial 1-5), Learning (trial 5-trial 1), Immediate Recall, Long Delay (30 minutes), \%Long Delay (percent of Maximum recalled); Rey, 1964],
Trail-Making Test A and B (Trails A and B; Reitan, 1955), Digit Span (Kaplan, Fein, Morris, \& Delis, 1991), FAS Test of phonemic verbal fluency (Borkowski, Benton, \& Spreen, 1967), Stroop Test [word (W) and color-word (CW); Golden, 1978] and Simple Reaction Time (Ballard et al., 2001). The DSST (Wechsler, 1944), a sensitive measure of processing speed, was administered along with test variants Symbol Copy (Kaplan et al., 1991) and Error Check (Joy, Fein, Kaplan, \& Freedman, 2000). Similar in procedure to the DSST, the two variants enable statistical delineation of DSST's cognitive and psychomotor task demands (Van der Elst, van Boxtel, van Breukelen, \& Jolles, 2006). In Symbol Copy, participants simply copy each symbol in the grid into an empty box directly below it as fast as possible, without consulting a coding key as in DSST, thereby isolating the DSST's graphomotor components. Error Check, conversely, involves scanning a completed DSST for errors in relation to a key and marking any with a pencil slash, thereby capturing visual scanning speed with minimal psychomotor demands. Cognitive scores other than the ACE-R and MMSE were not used for patient diagnoses.

\section{Data cleaning and analysis}

Following Little's Missing Completely at Random Test (MCAR) $\left(\chi^{2}[451]=480.3, p=.164\right)$, missing values were replaced using expectation-maximization (Statistical Package for the Social Sciences, V. 21; IBM SPSS Corp., 2013). Performance by group was compared using multivariate analyses of variance (MANOVA) for each cognitive domain followed by independent samples $t$ or Mann-Whitney $U$ tests, where appropriate. Effect sizes $(g)$ and $95 \%$ confidence intervals were bias-corrected (Hedges \& Olkin, 1985). As interindividual variation in performance can be great, MCI group scores were also computed as percentile rankings. Based on control group data, the percentage of each MCI group that scored at or below the 5th and 16th percentiles (1.65 and 1.0 $S D$ s below control means, respectively) was calculated. Variables differing significantly between MCI subtypes were also entered into a stepwise discriminant analysis to determine maximal differentiation between subtypes, excluding controls.

Domain composite scores were computed as average control-adjusted $z$-scores using representative outcome measures: executive function (FAS, Trails Ratio), verbal learning and memory (RAVLT Maximum and Short Delay) and visuospatial working memory (Corsi Blocks, VPT). Delayed memory was measured using RAVLT Long Delay (verbal delayed memory), and MTCF \%Recall (visuospatial delayed memory). A series of hierarchical multiple regressions (Enter method) run separately for MCI-AD/controls and MCI-LB/controls tested the mediating role of domaingeneral resources [executive function (FAS, Trails Ratio) and processing speed (DSST)] on domain-specific impairments (visuospatial and verbal learning and delayed memory) following a statistical procedure similar to Nebes et al. 
(2000). In all models, variance associated with age and estimated premorbid IQ [National Adult Reading Test (NART)] was initially accounted for in Step 1 and retained in subsequent models if significant. The proportion of diagnosisrelated variance in the domain-specific measures was first determined by entering "group" (control, MCI-AD or MCI-LB) as a predictor variable (Step 2). Next, models were run in which the domain-general variables of interest (processing speed and executive function) were entered iteratively at Step 2 before group (Step 3). The variance explained $\left(\Delta R^{2}\right)$ by group in these two models were then compared to calculate "group-associated variance explained" as the percent decrease in variance explained by group when it is entered at Step 3 (after processing speed or executive function) versus at Step 2. Group-associated variance explained therefore quantifies the domain-general predictor's (processing speed or executive function) mediation of the deficit due to MCI subtype status in a particular domainspecific measure (Nebes et al., 2000). Lastly, multiple regressions were run separately by group to predict DSST performance by its subtests (Symbol Copy, Error Check) and gross motor impairment (UPDRS).

\section{RESULTS}

One hundred and fifty four participants consented to the study, including $n=34$ controls with complete study data. Of $n=120 \mathrm{MCI}, n=16$ withdrew for various reasons (see online supplement fig. A1). Patients were diagnosed as probable MCI-LB $(n=44)$, MCI-AD $(n=39)$ or possible MCILB $(n=21$; as earlier, these were excluded from further analyses). MCI subtypes were equivalent on age, premorbid IQ and global cognition (MMSE). MCI-LB consisted of more males and showed greater severity on neuropsychiatric and functional measures (UPDRS, ESS, CAF, GDS, CIRS-G, IADL; see Table 1). Controls with abnormal MIBG $(n=2)$ or FP-CIT $(n=2)$ had normal clinical presentation, intact cognition and no other evidence of LB disease and were retained as healthy comparison subjects.

\section{Overall neuropsychological performance}

MANOVA of the four principal neuropsychological domains demonstrated statistically significant group differences: executive function, $F(12,170)=5.35, p<.001$; Wilk's $\Lambda=.527$, partial $\eta^{2}=.27$, processing speed, $F(12,166)=4.35$, $p<.001$; Wilk's $\Lambda=.579$, partial $\eta^{2}=.24$, visuospatial, $F(10,142)=3.36, p=.001 ;$ Wilk's $\Lambda=.654$, partial $\eta^{2}=.19$, and verbal learning and memory, $F(12,208)=5.32, p<.001$; Wilk's $\Lambda=.585$, partial $\eta^{2}=.24$. As expected, both MCI groups scored significantly below controls on all neuropsychological measures $(p s<.01)$ except Forward Digit Span, $\chi^{2}(2)=3.05, p=.218$, and Simple Reaction Time (SRT) $m u, \chi^{2}(2)=2.34, p=.310$. Effect sizes $(g)$ and $95 \%$ confidence intervals are presented in forest plots by domains
(Figure 1), showing MCI differences relative to controls and a pattern of divergence between MCI subtypes, particularly in processing speed and delayed recall tasks, both visuospatial and verbal. MCI-LB performed significantly worse than MCI-AD on Trails B $(p=.03, g=.55)$, Trails Ratio (A/B; $p=.04, g=.51)$, DSST $(p=.04, g=.48)$, Error Check $(p<.001, g=.75)$, and Stroop W $(p=.01, g=.54)$. MCI-AD performed significantly below MCI-LB on delayed verbal recall (RAVLT Long Delay, $p=.04, g=.37$; RAVLT $\%$ Long Delay, $p=.01, g=.48$ ) and delayed visuospatial recall (MTCF \%Recall, $p=.01, g=.62$ ).

The majority of MCI-LB patients performed at or below the 16th percentile (1SD or more below control means) on processing speed measures $(84.1 \%$ on Trails B, $88.6 \%$ DSST; see online supplement). In the visuospatial domain, percentile standings below the 5 th percentile were similar between subtypes in visuospatial working memory (VPT; MCI-LB 58.8\%, MCI-AD: 53.8\%) and figure drawing (MCI-LB: 40.9\%, MCI-AD: 48.7\%). However, a higher proportion of the MCI-AD group were impaired at the 5th percentile (1.65 SDs) in visuospatial delayed recall (MTCF \% Recall; MCI-AD: 43.6\%, MCI-LB: 29.5\%) and verbal delayed recall (RAVLT Long Delay; MCI-AD: $59.0 \%$, MCI-LB: $27.3 \%$ ).

Stepwise discriminant analysis excluding controls was applied to variables differing significantly between MCI subtypes. The model resulted in three variables in three steps: Error Check, $F(1,81)=9.91, p=.002$, MTCF \%Recall, $F(2,80)=9.84, p<.001$, and Trails Ratio, $F(3,79)=8.80$, $p<.001$. MTCF \%Recall had the highest standardized discriminant function coefficient (.74), followed by Error Check (-.62) and Trails Ratio (0.54). The canonical loadings were $\geq .30$ for all neuropsychological variables entered into the analysis except RAVLT Long Delay and \%Recall, suggesting a combination of visuospatial memory and executive-weighted cognitive processing. The discriminant function correctly classified subtype in $65.1 \%$ of all MCI cases, with $72.7 \%$ specificity and $56.4 \%$ sensitivity.

\section{Analysis of the hierarchical organization}

In controls, no significant relationships were observed in simple correlational assessment of the relationship between processing resources (DSST, executive function) and visuospatial and verbal variables (working memory and delayed memory; all $p \mathrm{~s}>.05)$. In MCI-LB, DSST was correlated with visuospatial working memory $\left(r_{s}=.524, p<.001\right)$ and delayed visuospatial memory $\left(r_{s}=.366, p=.015\right)$. In MCI$\mathrm{AD}$, executive function and visuospatial working memory were correlated, $\left(r_{s}=.331, p=.039\right)$, and DSST was correlated with all working and delayed memory measures: visuospatial working memory $\left(r_{s}=.394, p=.013\right)$, delayed visuospatial memory $\left(r_{s}=.405, p=.010\right)$, verbal learning and memory $\left(r_{s}=.444, p=.005\right)$, and delayed verbal memory $\left(r_{s}=.366, p=.022\right)$. 
Table 1. Demographics and clinical scales of MCI with Lewy bodies (MCI-LB; $n=44)$, MCI due to Alzheimer's disease (MCI-AD; $n=39$ ) and controls $(n=34)$, with significance $(p)$ of between-group comparisons of MCI subtypes

\begin{tabular}{|c|c|c|c|c|}
\hline & MCI-LB & MCI-AD & $p$ & Controls \\
\hline$n$ & 44 & 39 & & 34 \\
\hline Age, mean $(S D)$ & $74.9(6.3)$ & $76.2(7.6)$ & .39 & $74.2(7.5)$ \\
\hline Sex, males (females) & $37(7)$ & $16(23)$ & $<.001$ & $24(10)$ \\
\hline MMSE, mean $(S D)$ & $26.4(2.5)$ & $26.9(2.1)$ & .38 & $28.5(1.1)$ \\
\hline NART IQ, median (IQR) & $107.5(102.0-115.3)$ & $110.5(105.8-118.0)$ & .45 & $116.0(109.0-121.0)$ \\
\hline CIRS-G Total, mean (SD) & $8.5(4.0)$ & $6.4(3.2)$ & .02 & $5.29(3.3)$ \\
\hline UPDRS, median (IQR) & $21.0(10.5-35.5)$ & $12.0(4.0-23.0)$ & .01 & $5.0(2.0-8.0)$ \\
\hline NEVHI, median (IQR) & $.0(.0-5.5)$ & $.0(.0-1.0)$ & $<.001$ & $.0(.0-.0)$ \\
\hline ESS, median (IQR) & $9.0(6.0-10.3)$ & $4.0(2.0-9.0)$ & $<.001$ & $5.0(2.0-6.3)$ \\
\hline DCFS, median (IQR) & $8.0(6.8-10.3)$ & $6.5(5.0-8.3)$ & .01 & - \\
\hline CAF, median (IQR) & $3.0(.0-6.0)$ & $.0(.0-.8)$ & .01 & - \\
\hline GDS, median (IQR) & $4.0(2.0-9.0)$ & $3.0(1.0-5.0)$ & .04 & $1.0(.0-2.0)$ \\
\hline NPI Total, median (IQR) & $15.0(5.0-28.3)$ & $5.0(1.5-12.5)$ & $<.001$ & - \\
\hline NPI Total Distress, median (IQR) & $8.0(1.8-16.0)$ & $3.0(.5-6.5)$ & $<.001$ & - \\
\hline IADL, median (IQR) & $6.3(5.0-8.0)$ & $7.2(7.0-8.0)$ & $<.001$ & - \\
\hline CDR, median (IQR) & $.5(.5-.5)$ & $.5(.5-.5)$ & .05 & $.00(.0-.0)$ \\
\hline
\end{tabular}

Parametric data reported as mean and standard deviation $(S D)$, nonparametric data reported as median and interquartile range (IQR). Mann-Whitney $U$ tests, $t$, and Chi-squared tests used depending on the nature of the data. Bold denotes $p<.05$.

MMSE: Mini-Mental State Examination; NART: National Adult Reading Test; CIRS-G: Cumulative Illness Rating Scale for Geriatrics; UPDRS: Unified Parkinson's Disease Rating Scale (MDS Revision); NEVHI: North-East Visual Hallucinations Interview; ESS: Epworth Sleepiness Scale; DCFS: Diagnostic Cognitive Fluctuations Scale; CAF: Clinician Assessment of Fluctuation; GDS: Geriatric Depression Scale; NPI: Neuropsychiatric Inventory; IADL: Instrumental Activities of Daily Living Scale; CDR: Clinical Dementia Rating Scale.

Informant based scales MCI-AD $n=37$, NPI MCI-AD $n=36$; MCI-LB $n=40$, NEVHI MCI-LB $n=41$.
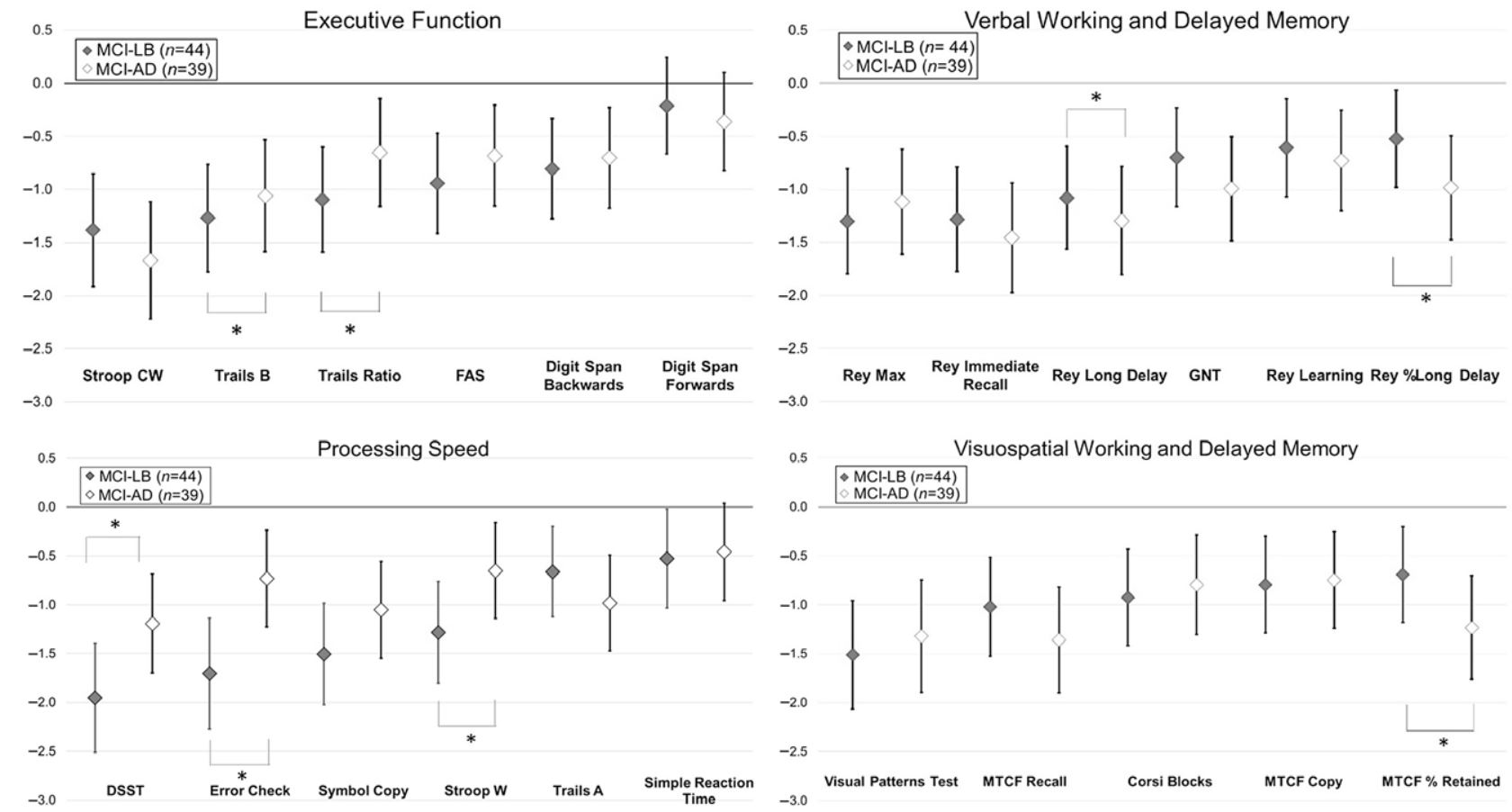

Fig. 1. Bias-adjusted effect sizes and 95\% CI (error bars) of control-centered ( $n=34)$ performance on neuropsychological tasks by MCI due to Alzheimer's disease (MCI-AD; $n=39$ ) and MCI with Lewy bodies (MCI-LB; $n=44$ ), plotted by domain (Executive Function, Processing Speed, Verbal Learning and Memory, Visuospatial Working and Delayed Memory). Significant differences between MCI subtypes indicated with asterisk $(*)$. 
Processing speed (DSST) was a stronger predictor of group-associated variance in verbal learning and memory, visuospatial working memory, and delayed visuospatial memory than executive function in both the MCI-LB/controls and MCI-AD/controls analyses (Table 2). In delayed verbal memory, processing speed explained $53.9 \%$ and $29.0 \%$ of group-associated variance in MCI-LB/controls and MCI$\mathrm{AD} /$ controls, respectively, but the relationship with executive function was not significant in either grouping $(p=.203$, $p=.082$, respectively). If entered after executive function, processing speed also explained additional unique variance in visuospatial working memory, verbal learning and memory, and delayed visuospatial memory scores in both groups. However, when order of entry was reversed, executive function did not add additional unique variance after accounting for processing speed. Processing speed's mediation of measures of visuospatial working memory (81.8\% of MCI-LB-associated variance explained) and delayed verbal memory (53.9\% of MCI-LB-associated variance explained) was stronger in MCI-LB than in MCI-AD (60.0\% and $29.0 \%$ of MCI-AD-associated variance explained, respectively).

\section{Processing speed and motor function}

After age and NART, UPDRS scores accounted for $18 \%$ of DSST score variance in MCI-AD $(F[1,34]=7.87, p=.008)$, but did not predict DSST scores in controls $\left(\Delta R^{2}=.00\right.$, $F[1,28]=.01, \quad p=.922), \quad$ nor $\mathrm{MCI}-\mathrm{LB} \quad\left(\Delta R^{2}=.00\right.$, $F[1,36]=.01, p=.920)$. Error Check, which isolates visual scanning efficiency, explained the largest amount of DSST score variance in all three groups (controls: $75 \%, F(1,29)=$ 47.50, $p<.001$; MCI-LB: 77\%, $F(1,38)=106.25, p<.001$; MCI-AD: $76 \%, F(1,33)=119.74, p<.001)$, and explained an additional $3 \%$ of unique variance after accounting for graphomotor speed (Symbol Copy) in controls, $15 \%$ in MCI-LB $(p<.001)$, and $20 \%$ in MCI-AD $(p<.001)$. Graphomotor speed explained less variance in controls $(11 \%, F[1,29]=6.21, p=.019)$ than MCI subtypes (MCILB: $49 \%, F[1,38]=56.68, p<.001$; MCI-AD: $40 \%, F[1,33]=$ $34.34, p<.001)$. However, after accounting for visual scanning, graphomotor speed no longer significantly predicted DSST in controls $\left(\Delta R^{2}=.03, F[1,28]=4.07, p=.053\right)$ and explained only small additional variance in MCI-LB $\left(\Delta R^{2}=.04, \quad F[1,37]=8.77, \quad p=.005\right) \quad$ and $\mathrm{MCI}-\mathrm{AD}$ $\left(\Delta R^{2}=.03, F[1,32]=4.43, p=.043\right)$.

\section{DISCUSSION}

The present study aimed to characterize the neuropsychological profile of MCI-LB compared to MCI-AD and healthy older people, and to identify if impairments in MCI are related to deficits in domain-general cognitive resources, such as executive dysfunction or slowed processing speed. Both MCI subtypes scored significantly lower than healthy controls on all neuropsychological measures except immediate memory and simple reaction time. However, divergence between MCI groups was also evident, despite having a similar level of global cognitive impairment (MMSE and CDR). The MCI-LB group was impaired relative to the MCI-AD group on measures of cognitive processing speed and executive function, in line with previous work (Ciafone et al., 2020), but generally had similar levels of visuospatial dysfunction as MCI-AD, contrary to expectations (Cagnin et al., 2015; Donaghy, Taylor, et al., 2018).

Examination of the hierarchical neuropsychological organization revealed a profile in MCI that was not evident in healthy older adults. Working memory impairment and multidomain amnesia in MCI were substantially related to slowed speed of processing, as measured by the DSST, a well-established test sensitive to neurological dysfunction and validated in a variety of populations (Van der Elst et al., 2006). Processing speed, a distinct yet interrelated concept with executive function, is the domain-general speed of execution of basic cognitive functions. This process thereby limits completion of time-sensitive actions (e.g., memory formation, which necessitates information processing before working memory decay; Luszcz \& Bryan, 1999; Nebes et al., 2000). Processing speed was also a better explanatory factor than the executive function composite (verbal fluency and Trails Ratio) in both subtypes. In MCI-LB, executive function did explain a significant proportion of verbal learning and memory and delayed memory impairment, but this relationship was completely accounted for by differences in processing speed. This mediating role of speed of processing could be argued to be due to the motor impairments associated with MCI-LB. However, subanalyses of the DSST indicated that this measure was not related to motor impairment (UPDRS) in MCI-LB and was more strongly related to the cognitive aspect of the task (visual scanning) than to slowed graphomotor speed, in line with previous reports in Lewy body dementia (Firbank, O'Brien, \& Taylor, 2018) and PD (Johnson et al., 2004). LB disease is associated with substantial deficits in the cholinergic system (Ballard et al., 2001), key to the attentional abilities involved in information processing. Presynaptic dysfunction driven by alpha-synuclein aggregates is present even at early stages of LB disease (Kramer \& Schulz-Schaeffer, 2007; SchulzSchaeffer, 2010), and our results similarly demonstrate processing speed slowing in the MCI phase. Both MCI subtypes showed a hierarchical structure of performance predicted by their speed of processing, although the magnitude of the effect was notably smaller in MCI-AD than in MCI-LB in the case of visuospatial working memory and delayed verbal memory. Taken together, while MCI-LB had significantly slower processing speed than MCI-AD, it may nevertheless be a feature common to neurodegenerative diseases.

Given the lack of evidence of poorer visuospatial function in MCI-LB relative to MCI-AD in the present study, future work should investigate the trajectory of visuospatial decline in MCI. In a longitudinal analysis of a previous cohort, we found that visuospatial function declined more rapidly in MCI-LB (Hamilton et al., 2021). This finding was also reported by another group in DLB compared with AD (Smirnov et al., 


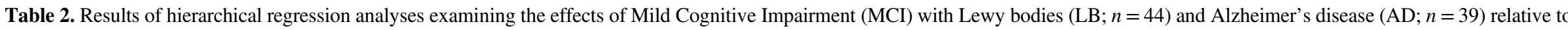
healthy control subjects $(n=34)$, and the processing speed and executive function measures on neuropsychological domain performance

\begin{tabular}{|c|c|c|c|c|c|c|}
\hline & & \multirow{2}{*}{$\begin{array}{l}\text { Group-Associated } \\
\text { Variance }\end{array}$} & Processing Speed & Executive Function & $\begin{array}{l}\text { Processing Speed after } \\
\text { Executive Function }\end{array}$ & $\begin{array}{l}\text { Executive Function after } \\
\text { Processing Speed }\end{array}$ \\
\hline & & & \multicolumn{2}{|c|}{$\%$ Group-Associated Variance Explained $\left(\Delta R^{2}\right)$} & \multicolumn{2}{|c|}{ Additional Variance Explained $\left(\Delta R^{2}\right)$} \\
\hline \multirow[t]{4}{*}{$\begin{array}{l}\text { MCI-LB \& } \\
\text { Controls }\end{array}$} & $\begin{array}{l}\text { Verbal Learning and } \\
\text { Memory }\end{array}$ & $\begin{array}{c}\Delta R^{2}=.21 \\
F(3,71)=26.71 \\
\quad p<.001\end{array}$ & $\begin{array}{c}\mathbf{6 6 . 7 \%}, \\
\Delta R^{2}=.07, F(4,70)=9.24, \\
p=.003\end{array}$ & $\begin{array}{c}\mathbf{3 3 . 3 \%} \\
\Delta R^{2}=.11, F(4,70)=8.31 \\
p=.005\end{array}$ & $\begin{array}{c}\mathbf{8 . 0 \%} \\
\Delta R^{2}=.08, F(4,70)=9.69 \\
p=.003\end{array}$ & n.s., $p=.150$ \\
\hline & $\begin{array}{l}\text { Visuospatial Working } \\
\text { Memory }\end{array}$ & $\begin{array}{c}\Delta R^{2}=.33 \\
F(3,71)=38.98 \\
\quad p<.001\end{array}$ & $\begin{array}{c}\mathbf{8 1 . 8 \%}, \\
\Delta R^{2}=.06, F(4,70)=7.73, \\
p=.007\end{array}$ & $\begin{array}{c}\mathbf{3 6 . 4 \%} \\
\Delta R^{2}=.14 \\
F(4,70)=12.84 \\
p<.001\end{array}$ & $\begin{array}{c}\mathbf{2 1 . 0 \%} \\
\Delta R^{2}=.21, F(4,70)=24.81 \\
p<.001\end{array}$ & $n . s ., p=.120$ \\
\hline & $\begin{array}{l}\text { Delayed Verbal } \\
\text { Memory }\end{array}$ & $\begin{array}{c}\Delta R^{2}=.13 \\
F(3,71)=13.13 \\
\quad p=.001\end{array}$ & $\begin{array}{c}\mathbf{5 3 . 9 \%} \\
\Delta R^{2}=.07, F(4,70)=6.31 \\
p=.014\end{array}$ & n.s. $(.203)$ & - & - \\
\hline & $\begin{array}{l}\text { Delayed Visuospatial } \\
\text { Memory }\end{array}$ & $\begin{array}{c}\Delta R^{2}=.16 \\
F(3,71)=17.90 \\
\quad p<.001\end{array}$ & $\begin{array}{c}\mathbf{6 2 . 5 \%} \\
\Delta R^{2}=.11 \\
F(4,70)=11.50 \\
p=.001\end{array}$ & $\begin{array}{c}\mathbf{4 3 . 8 \%} \\
\Delta R^{2}=.10, F(4,66)=9.44 \\
p=.003\end{array}$ & $\begin{array}{c}\mathbf{5 . 0 \%} \\
\Delta R^{2}=.05, F(4,66)=5.44 \\
p=.023\end{array}$ & n.s., $p=.063$ \\
\hline \multirow[t]{4}{*}{$\begin{array}{l}\text { MCI-AD \& } \\
\text { Controls }\end{array}$} & $\begin{array}{l}\text { Verbal Learning and } \\
\text { Memory }\end{array}$ & $\begin{array}{c}\Delta R^{2}=.30 \\
F(3,67)=34.71 \\
\quad p<.001\end{array}$ & $\begin{array}{c}\mathbf{6 3 . 3 \%} \\
\Delta R^{2}=.11 \\
F(4,66)=14.08, \\
p<.001\end{array}$ & $\begin{array}{c}\mathbf{2 6 . 7 \%} \\
\Delta R^{2}=.10, F(4,66)=8.58 \\
p=.005\end{array}$ & $\begin{array}{c}\mathbf{9 . 0 \%} \\
\Delta R^{2}=.09, F(4,66)=9.69 \\
p=.003\end{array}$ & n.s., $p=.283$ \\
\hline & $\begin{array}{l}\text { Visuospatial Working } \\
\text { Memory }\end{array}$ & $\begin{array}{c}\Delta R^{2}=.30 \\
F(3,67)=38.12 \\
\quad p<.001\end{array}$ & $\begin{array}{c}\mathbf{6 0 . 0 \%} \\
\Delta R^{2}=.12 \\
F(4,66)=17.42 \\
p<.001\end{array}$ & $\begin{array}{c}\mathbf{4 0 . 0 \%} \\
\Delta R^{2}=.18 \\
F(4,66)=12.44 \\
\quad p=.001\end{array}$ & $\begin{array}{c}\mathbf{8 . 0 \%} \\
\Delta R^{2}=.08, F(4,66)=9.17 \\
p=.003\end{array}$ & n.s., $p=.154$ \\
\hline & $\begin{array}{l}\text { Delayed Verbal } \\
\text { Memory }\end{array}$ & $\begin{array}{c}\Delta R^{2}=.29 \\
F(3,67)=30.46 \\
\quad p<.001\end{array}$ & $\begin{array}{c}\mathbf{2 9 . 0 \%} \\
\Delta R^{2}=.26 \\
F(4,66)=21.49 \\
p<.001\end{array}$ & n.s., $p=.082$ & - & - \\
\hline & $\begin{array}{l}\text { Delayed Visuospatial } \\
\text { Memory }\end{array}$ & $\begin{array}{c}\Delta R^{2}=.31 \\
F(3,67)=34.06 \\
\quad p<.001\end{array}$ & $\begin{array}{c}\mathbf{5 4 . 8 \%} \\
\Delta R^{2}=.23 \\
F(4,66)=22.65 \\
p<.001\end{array}$ & $\begin{array}{c}\mathbf{3 2 . 2 \%} \\
\Delta R^{2}=.16 \\
F(4,66)=14.36 \\
p<.001\end{array}$ & $\begin{array}{c}\mathbf{9 . 0 \%} \\
\Delta R^{2}=.09, F(4,66)=9.26 \\
p=.003\end{array}$ & n.s., $p=.134$ \\
\hline
\end{tabular}

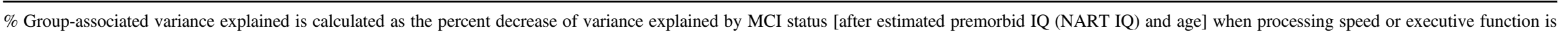

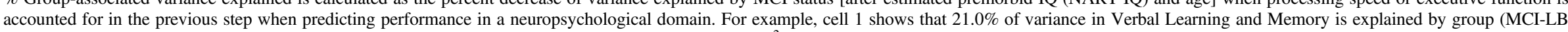

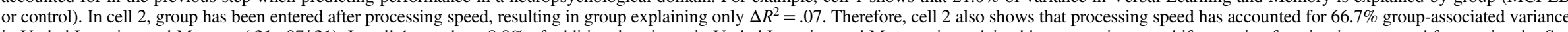

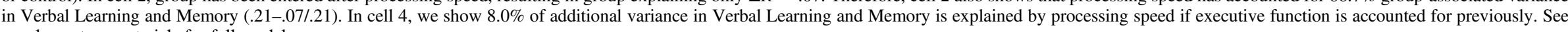
supplementary materials for full model. 
2020), although not by van de Beek et al. (2020) in MCI-LB versus MCI-AD. Complex visual hallucinations also predict more rapid decline in MCI-LB (Hamilton et al., 2020). Visuospatial function is suggested to have greater dependency on the executive system than verbal function, indicating a lack of symmetry in multicomponential models of working memory (Thompson et al., 2006). While exploratory, it is possible that pronounced visuospatial dysfunction may only become apparent in DLB as a secondary effect as executive dysfunction worsens over the disease course. Executive function is a multifaceted construct, empirically delineated to include processes like set-shifting, updating, and inhibition (Miyake et al., 2000). The executive function measures employed in the present study (Trails and phonemic verbal fluency) are primarily dependant on set-shifting (Snyder, Miyake, \& Hankin, 2015), noted as impaired in MCI-LB (Cagnin et al., 2015; Jicha et al., 2010). While executive functions share a common mechanism (Snyder, 2013), more extensive testing could capture the construct more fully. Furthermore, a limitation of the present study is the omission of additional basic visuospatial working memory measures, such as line orientation tests, that may be less dependent on executive abilities.

Delayed recall conditions, whether for verbal or visuospatial (MTCF) stimuli, revealed amnestic impairment in MCI-AD, suggesting a profile similar to advanced AD. For example, $59 \%$ of MCI-AD patients scored at or below the 5 th percentile of controls on tests of delayed verbal memory. However, more than a quarter of MCI-LB patients also demonstrated delayed verbal memory deficits below the 5th percentile of controls. Moreover, the stepwise discriminant analysis resulted in a model omitting verbal memory as predictive of group membership. Previous work by Ferman et al. (2013) showed that, although single-domain amnestic MCI rarely progresses to DLB, memory impairment is common in prodromal DLB patients with attention and/or visuospatial dysfunction (24.5\%). Therefore, despite significantly poorer performance in MCI-AD at a group level, memory impairments can present in clinically-defined MCI-LB patients (Ferman et al., 2013; Kemp et al., 2017). The extent to which an amnestic presentation in LB disease is attributable to cooccurring $\mathrm{AD}$ neuropathology remains debated (Ferman et al., 2013) but it is a frequent presentation, with a recent analysis of 670 brains finding over $11 \%$ of people with dementia had both high grade AD and DLB (McAleese et al., 2021). However, recent work failed to find differences in neuropsychological or neuropsychiatric profile and cognitive fluctuations between amyloid-positive and amyloidnegative DLB cases using PET imaging (Donaghy, Firbank, et al., 2018). The phenomenon of multiple pathologies and the possible interactions of concurrent AD and DLB pathology in many people with dementia challenge attempts to delineate clear, aetiologically-specific neuropsychological profiles, with less than a quarter of cases in the above analysis having pure pathology (McAleese et al., 2021). However, the present study benefited from the utilization of two biomarkers of LB disease and annually-repeated neuropsychological and clinical assessments. While it is a limitation of our study that we were not able to incorporate AD biomarkers such as amyloid imaging and cerebrospinal fluid, the fact we rigorously excluded vascular, Lewy body, and frontotemporal aetiologies means our MCI-AD group would predominantly have been people with $\mathrm{AD}$. In general, the discriminant utility of neuropsychological assessment used in isolation has been shown to be limited in MCI (Donaghy et al., 2020), emphasizing the importance of continued validation of the proposed MCI-LB biomarkers.

In summary, the present study suggests that MCI-LB and MCI-AD display cognitive impairments in line with the advanced stages of DLB and AD, respectively, although there was considerable overlap in their general neuropsychological profile. MCI-LB was characterized by executive dysfunction and slowed speed of processing, while MCI-AD displayed an amnestic profile. The assessment of the findings within their hierarchical framework indicated that deficits in higher-order cognitive activities in both MCI subtypes were mediated by processing speed, a profile which was strongest in MCI-LB and entirely absent in healthy controls.

\section{SUPPLEMENTARY MATERIAL}

To view supplementary material for this article, please visit https://doi.org/10.1017/S1355617721001181

\section{FINANCIAL SUPPORT}

This study was supported by a major project research grant from Alzheimer's Research UK (ARUK-PG2015-13); a doctoral studentship from the Medical Research Council (J.C.); ligand for the FP-CIT scans provided by GE Healthcare; an Alzheimer's Society healthcare professional Fellowship (G.R.); infrastructure and support for authors based at Newcastle by the National Institute for Health Research (NIHR) Newcastle Biomedical Research Centre, a partnership between Newcastle upon Tyne Hospitals NHS Foundation Trust and Newcastle University; and the NIHR Cambridge Biomedical Research Centre (J.O.B).

\section{CONFLICTS OF INTEREST}

AT has received support from GE Healthcare for investigator led research including the provision of FP-CIT in this study. Outside of this work JPT has acted as a consultant for Kyowa Kirin, Heptares Sosei, and received grant funding from Heptares Sosei and speaker fees from GE Healthcare. GR and GP have received honoraria from GE Healthcare for delivering educational workshops on FP-CIT imaging. Outside of this work JOB has acted as a consultant for TauRx, Axon, Eisai, Roche, and GE Healthcare and received 
grant funding from Alliance Medical and Merck. RD has received an educational travel grant from Britannia outside of this work. JC, PCD, CAH, SL, SC, MF, LA report no financial relationships with commercial interests.

\section{REFERENCES}

Albert, M. S., DeKosky, S. T., Dickson, D., Dubois, B., Feldman, H. H., Fox, N. C., ... Phelps, C. H. (2011). The diagnosis of mild cognitive impairment due to Alzheimer's disease: Recommendations from the National Institute on AgingAlzheimer's Association workgroups on diagnostic guidelines for Alzheimer's disease. Alzheimer's \& Dementia, 7(3), 270-279.

Arnáiz, E., \& Almkvist, O. (2003). Neuropsychological features of mild cognitive impairment and preclinical Alzheimer's disease. Acta Neurologica Scandinavica, 107(s179), 34-41.

Ballard, C., O’Brien, J., Gray, A., Cormack, F., Ayre, G., Rowan, E., ... Walker, M. (2001). Attention and fluctuating attention in patients with dementia with Lewy bodies and Alzheimer disease. Archives of Neurology, 58(6), 977-982.

Borkowski, J. G., Benton, A. L., \& Spreen, O. (1967). Word fluency and brain damage. Neuropsychologia, 5(2), 135-140.

Breitve, M. H., Chwiszczuk, L. J., Brønnick, K., Hynninen, M. J., Auestad, B. H., Aarsland, D., \& Rongve, A. (2018). A longitudinal study of neurocognition in dementia with Lewy bodies compared to Alzheimer's disease. Frontiers in Neurology, 9, 124.

Brown, L. A., \& Wesley, R. W. (2013). Visual working memory is enhanced by mixed strategy use and semantic coding. Journal of Cognitive Psychology, 25(3), 328-338.

Cagnin, A., Bussè, C., Gardini, S., Jelcic, N., Guzzo, C., Gnoato, F., ... Caffarra, P. (2015). Clinical and cognitive phenotype of mild cognitive impairment evolving to dementia with Lewy bodies. Dementia and Geriatric Cognitive Disorders Extra, 5(3), 442449. doi: 10.1159/000441184

Ciafone, J., Little, B., Thomas, A. J., \& Gallagher, P. (2020). The neuropsychological profile of Mild Cognitive Impairment in Lewy body dementias. Journal of the International Neuropsychological Society, 26(2), 210-225.

Corp., I. (2013). IBM SPSS Statistics for Windows (Version Version 21.0). Armonk, NY: IBM Corp.

Corsi, P. M. (1972). Human memory and the medial temporal region of the brain. Dissertation Abstracts International, 34(819B).

Cummings, J. L., Mega, M., Gray, K., Rosenberg-Thompson, S., Carusi, D. A., \& Gornbein, J. (1994). The neuropsychiatric inventory: Comprehensive assessment of psychopathology in dementia. Neurology, 44(12), 2308-2308.

D’Ath, P., Katona, P., Mullan, E., Evans, S., \& Katona, C. (1994). Screening, detection and management of depression in elderly primary care attenders. I: The acceptability and performance of the 15 item Geriatric Depression Scale (GDS15) and the development of short versions. Family Practice, 11(3), 260-266.

Della Sala, S., Baddeley, A. D., Gray, C., \& Wilson, L. (1997). Visual Patterns Test: VPT. Harcourt.

Donaghy, P. C., Ciafone, J., Durcan, R., Hamilton, C. A., Barker, S., Lloyd, J., ... Taylor, J.-P. (2020). Mild cognitive impairment with Lewy bodies: Neuropsychiatric supportive symptoms and cognitive profile. Psychological Medicine, 1-9.

Donaghy, P. C., Firbank, M. J., Thomas, A. J., Lloyd, J., Petrides, G., Barnett, N., ... O'Brien, J. T. (2018). Clinical and imaging correlates of amyloid deposition in dementia with Lewy bodies. Movement Disorders.
Donaghy, P. C., O’Brien, J. T., \& Thomas, A. J. (2015). Prodromal dementia with Lewy bodies. Psychological Medicine, 45(2), 259_ 268. doi: 10.1017/S0033291714000816

Donaghy, P. C., Taylor, J. P., O’Brien, J. T., Barnett, N., Olsen, K., Colloby, S. J., ... Thomas, A. J. (2018). Neuropsychiatric symptoms and cognitive profile in mild cognitive impairment with Lewy bodies. Psychological Medicine, 48(14), 2384-2390. doi: 10.1017/S0033291717003956

Ferman, T. J., Smith, G. E., Kantarci, K., Boeve, B. F., Pankratz, V. S., Dickson, D. W., ... Petersen, R. C. (2013). Nonamnestic mild cognitive impairment progresses to dementia with Lewy bodies. Neurology, 81(23), 2032-2038. doi: 10.1212/01.wnl. 0000436942.55281 .47

Firbank, M. J., O’Brien, J. T., Durcan, R., Allan, L. M., Barker, S., Ciafone, J., ... Lloyd, J. (2020). Mild cognitive impairment with Lewy bodies: Blood perfusion with arterial spin labelling. Journal of Neurology, 1-11.

Firbank, M. J., O’Brien, J. T., \& Taylor, J. P. (2018). Long reaction times are associated with delayed brain activity in Lewy body dementia. Human Brain Mapping, 39(2), 633-643.

Folstein, M., Folstein, S., \& McHugh, P. (1975). Mini-mental state examination: A practical method for grading the cognitive state of patients for physicians. Journal of Psychiatric Research, 12, 128-198.

Goetz, C. G., Tilley, B. C., Shaftman, S. R., Stebbins, G. T., Fahn, S., Martinez-Martin, P., ... Dodel, R. (2008). Movement Disorder Society-sponsored revision of the Unified Parkinson's Disease Rating Scale (MDS-UPDRS): Scale presentation and clinimetric testing results. Movement Disorders: Official Journal of the Movement Disorder Society, 23(15), 2129-2170.

Golden, C. J. (1978). Stroop colour and word test. Age, 15, 90.

Hamilton, C. A., Matthews, F. E., Donaghy, P. C., Taylor, J.-P., O’Brien, J. T., Barnett, N., ... McKeith, I. G. (2021). Cognitive decline in mild cognitive impairment with Lewy bodies or Alzheimer disease: A prospective cohort study. The American Journal of Geriatric Psychiatry, 29(3), 272-284.

Hamilton, C. A., Matthews, F. E., Donaghy, P. C., Taylor, J.-P., O'Brien, J. T., Barnett, N., ... Thomas, A. J. (2020). Prospective predictors of decline V. Stability in mild cognitive impairment with Lewy bodies or Alzheimer's disease. Psychological Medicine, 1-9. doi: 10.1017/S0033291720001130

Hedges, L., \& Olkin, I. (1985). Statistical Methods for MetaAnalysis. New York: Academic Press.

Hemminghyth, M. S., Chwiszczuk, L. J., Rongve, A., \& Breitve, M. H. (2020). The cognitive profile of mild cognitive impairment due to dementia with Lewy bodies-An updated review. Frontiers in Aging Neuroscience, 12.

Howard, E., Irwin, D. J., Rascovsky, K., Nevler, N., Shellikeri, S., Tropea, T. F., ... Siderowf, A. (2021). Cognitive profile and markers of Alzheimer disease-Type pathology in patients with Lewy body dementias. Neurology, 96(14), e1855-e1864.

Hubley, A. M., \& Tremblay, D. (2002). Comparability of total score performance on the Rey-Osterrieth complex figure and a modified Taylor complex figure. Journal of Clinical and Experimental Neuropsychology, 24(3), 370-382.

Hughes, C. P., Berg, L., Danziger, W., Coben, L. A., \& Martin, R. L. (1982). A new clinical scale for the staging of dementia. The British Journal of Psychiatry, 140(6), 566-572.

Jicha, G., Schmitt, F., Abner, E., Nelson, P., Cooper, G., Smith, C., \& Markesbery, W. (2010). Prodromal clinical manifestations of neuropathologically confirmed Lewy body disease. Neurobiology of Aging, 31(10), 1805-1813. 
Johns, M. W. (1991). A new method for measuring daytime sleepiness: The Epworth sleepiness scale. Sleep, 14(6), 540-545.

Johnson, A. M., Almeida, Q. J., Stough, C., Thompson, J. C., Singarayer, R., \& Jog, M. S. (2004). Visual inspection time in Parkinson's disease: Deficits in early stages of cognitive processing. Neuropsychologia, 42(5), 577-583.

Joy, S., Fein, D., Kaplan, E., \& Freedman, M. (2000). Speed and memory in WAIS-R-NI Digit Symbol performance among healthy older adults. Journal of the International Neuropsychological Society, 6(07), 770-780.

Kaplan, E., Fein, D., Morris, R., \& Delis, D. (1991). WAIS-R NI Manual. San Antonio: Psychological Corporation.

Kemp, J., Philippi, N., Phillipps, C., Demuynck, C., Albasser, T., Martin-Hunyadi, C., ... Blanc, F. (2017). Cognitive profile in prodromal dementia with Lewy bodies. Alzheimer's Research \& Therapy, 9(1), 19.

Kramer, M. L., \& Schulz-Schaeffer, W. J. (2007). Presynaptic $\alpha$-synuclein aggregates, not Lewy bodies, cause neurodegeneration in dementia with Lewy bodies. Journal of Neuroscience, 27(6), 1405-1410.

Lange, K. L., Bondi, M. W., Salmon, D. P., Galasko, D., Delis, D. C., Thomas, R. G., \& Thal, L. J. (2002). Decline in verbal memory during preclinical Alzheimer's disease: Examination of the effect of APOE genotype. Journal of the International Neuropsychological Society, 8(07), 943-955.

Lawton, M., Brody, E., \& Médecin, U. (1969). Instrumental activities of daily living (IADL). The Gerontologist, 9, 179-186.

Lee, D. R., McKeith, I., Mosimann, U., Ghosh-Nodial, A., Grayson, L., Wilson, B., \& Thomas, A. J. (2014). The dementia cognitive fluctuation scale, a new psychometric test for clinicians to identify cognitive fluctuations in people with dementia. The American Journal of Geriatric Psychiatry, 22(9), 926-935.

Lemstra, A., De Beer, M., Teunissen, C., Schreuder, C., Scheltens, P., Van Der Flier, W., \& Sikkes, S. (2017). Concomitant AD pathology affects clinical manifestation and survival in dementia with Lewy bodies. Journal of Neurology, Neurosurgery \& Psychiatry, 88(2), 113-118.

Logie, R. H. (2011). The functional organization and capacity limits of working memory. Current directions in Psychological Science, 20(4), 240-245.

Luszcz, M. A., \& Bryan, J. (1999). Toward understanding age-related memory loss in late adulthood. Gerontology, 45(1), 2-9.

MacDonald, S. W. S., Hultsch, D. F., Strauss, E., \& Dixon, R. A. (2003). Age-related slowing of digit symbol substitution revisited: What do longitudinal age changes reflect? The Journals of Gerontology Series B: Psychological Sciences and Social Sciences, 58(3), P187-P194.

Martin, A., Brouwers, P., Cox, C., \& Fedio, P. (1985). On the nature of the verbal memory deficit in Alzheimer's disease. Brain and Language, 25(2), 323-341.

McAleese, K. E., Colloby, S. J., Thomas, A. J., Al-Sarraj, S., Ansorge, O., Neal, J., ... Attems, J. (2021). Concomitant neurodegenerative pathologies contribute to the transition from mild cognitive impairment to dementia. Alzheimer's \& Dementia, 1(13). doi: 10.1002/alz.12291

McKeith, I., Ballard, C., Perry, R., Ince, P., O’brien, J., Neill, D., . . . Thompson, P. (2000). Prospective validation of consensus criteria for the diagnosis of dementia with Lewy bodies. Neurology, 54(5), 1050-1058.

McKeith, I., O’Brien, J., Walker, Z., Tatsch, K., Booij, J., Darcourt, J., ... Volterrani, D. (2007). Sensitivity and specificity of dopamine transporter imaging with 123 I-FP-CIT SPECT in dementia with Lewy bodies: A phase III, multicentre study. The Lancet Neurology, 6(4), 305-313.

McKeith, I. G., Ferman, T. J., Thomas, A. J., Blanc, F., Boeve, B. F., Fujishiro, H., ... Postuma, R. B. (2020). Research criteria for the diagnosis of prodromal dementia with Lewy bodies. Neurology.

Miller, M. D., Paradis, C. F., Houck, P. R., Mazumdar, S., Stack, J. A., Rifai, A. H., ... Reynolds III, C. F. (1992). Rating chronic medical illness burden in geropsychiatric practice and research: Application of the Cumulative Illness Rating Scale. Psychiatry Research, 41(3), 237-248.

Mioshi, E., Dawson, K., Mitchell, J., Arnold, R., \& Hodges, J. R. (2006). The Addenbrooke's Cognitive Examination Revised (ACE-R): A brief cognitive test battery for dementia screening. International Journal of Geriatric Psychiatry: A Journal of the Psychiatry of Late Life and Allied Sciences, 21(11), 1078-1085.

Miyake, A., Friedman, N. P., Emerson, M. J., Witzki, A. H., Howerter, A., \& Wager, T. D. (2000). The unity and diversity of executive functions and their contributions to complex "frontal lobe" tasks: A latent variable analysis. Cognitive Psychology, 4l(1), 49-100.

Mosimann, U. P., Collerton, D., Dudley, R., Meyer, T. D., Graham, G., Dean, J. L., .. Clarke, M. P. (2008). A semistructured interview to assess visual hallucinations in older people. International Journal of Geriatric Psychiatry: A Journal of the Psychiatry of Late Life and Allied Sciences, 23(7), 712-718.

Nebes, R. D., Butters, M. A., Mulsant, B. H., Pollock, B. G., Zmuda, M. D., Houck, P. R., \& Reynolds, C. F. (2000). Decreased working memory and processing speed mediate cognitive impairment in geriatric depression. Psychological Medicine, 30(03), 679-691.

Nelson, H. E., \& Willison, J. (1991). National adult reading test (NART): Nfer-Nelson Windsor.

Reitan, R. M. (1955). The relation of the trail making test to organic brain damage. Journal of Consulting Psychology, 19(5), 393.

Rey, A. (1964). L'examen clinique en psychologie. Paris: Presses Universitaires de France.

Roberts, G., Donaghy, P. C., Lloyd, J., Durcan, R., Petrides, G., Colloby, S. J., ... Firbank, M. (2020). Accuracy of dopaminergic imaging as a biomarker for mild cognitive impairment with Lewy bodies. The British Journal of Psychiatry, 1-7.

Schulz-Schaeffer, W. J. (2010). The synaptic pathology of $\alpha$-synuclein aggregation in dementia with Lewy bodies, Parkinson's disease and Parkinson's disease dementia. Acta Neuropathologica, 120(2), 131-143.

Smirnov, D. S., Galasko, D., Edland, S. D., Filoteo, J. V., Hansen, L. A., \& Salmon, D. P. (2020). Cognitive decline profiles differ in Parkinson disease dementia and dementia with Lewy bodies. Neurology, 94(20), e2076-e2087.

Snyder, H. R. (2013). Major depressive disorder is associated with broad impairments on neuropsychological measures of executive function: A meta-analysis and review. In: American Psychological Association.

Snyder, H. R., Miyake, A., \& Hankin, B. L. (2015). Advancing understanding of executive function impairments and psychopathology: Bridging the gap between clinical and cognitive approaches. Frontiers in Psychology, 6, 328.

Thomas, A. J., Donaghy, P., Roberts, G., Colloby, S. J., Barnett, N. A., Petrides, G., ... McKeith, I. (2019). Diagnostic accuracy of dopaminergic imaging in prodromal dementia with Lewy bodies. Psychological Medicine, 49(3), 396-402.

Thompson, J. M., Hamilton, C. J., Gray, J. M., Quinn, J. G., Mackin, P., Young, A. H., \& Ferrier, I. N. (2006). Executive and 
visuospatial sketchpad resources in euthymic bipolar disorder: Implications for visuospatial working memory architecture. MEMORY, 14(4), 437-451.

van de Beek, M., van Steenoven, I., van der Zande, J. J., Barkhof, F., Teunissen, C. E., van der Flier, W. M., \& Lemstra, A. W. (2020). Prodromal dementia with Lewy bodies: Clinical characterization and predictors of progression. Movement Disorders, 35(5), 859-867.

Van der Elst, W., van Boxtel, M. P. J., van Breukelen, G. J. P., \& Jolles, J. (2006). The Letter Digit Substitution Test: Normative data for 1,858 healthy participants aged 24-81 from the
Maastricht Aging Study (MAAS): Influence of age, education, and sex. Journal of Clinical and Experimental Neuropsychology, 28(6), 998-1009.

Walker, M., Ayre, G., Cummings, J., Wesnes, K., McKeith, I., O'brien, J., \& Ballard, C. (2000). The clinician assessment of fluctuation and the one day fluctuation assessment scale: Two methods to assess fluctuating confusion in dementia. The British Journal of Psychiatry, 177(3), 252-256.

Warrington, E. K. (1997). The graded naming test: A restandardisation. Neuropsychological Rehabilitation, 7(2), 143-146.

Wechsler, D. (1944). The measurement of adult intelligence. 